\title{
Performance of Integrated Data Warehouse Architecture for Financial Institutes with Security Levels
}

\author{
Vaibhav R. Bhedi ${ }^{1}$, Shrinivas P. Deshpande ${ }^{2}$, Ujwal A. Lanjewar ${ }^{3}$ \\ ${ }^{I}$ Assistant Professor, MCA, VMV Commerce, JMT Arts and JJP Science College, Nagpur, India \\ ${ }^{2}$ Associate Professor, P.G.D.C.S.T, D.C.P.E, H.V.P.M, Amravati, India \\ ${ }^{3}$ Assistant Professor, MCA, VMV Commerce, JMT Arts and JJP Science College, Nagpur, India
}

\begin{abstract}
The "Integrated Data Warehouse Architecture for Financial Institutes with Security Levels" is proposed data warehouse architecuture for overall Indian Finacial System [1][2][3][4][5]. The performance of architecture has been measure in SQL Server Management Studio by observing results[5]. The paper focus on the performance of architecture results and proved the executed queries have been given satisfactory performance. The performance of result is functionally correct because it behaves according to its stated functional specifications and gets the result according to our proposed expectation because it does not produce dangerous or costly failures [6]. It is used in a reasonable manner, that is, in a manner that a typical user expects is normal [7].
\end{abstract}

Keywords: ATM, BUID

\section{Introduction}

The proposed integrated data warehouse for financial institute with security levels has integrated with "Financial Transaction Model" [2], "Data Warehouse Architecture for Financial Institute" [3] and "Security Model" [4]. In financial transaction model, BUID will directly interact with channel like ATM, Kiosk, Funds, Call center, portal, internet, mobile etc to perform financial transaction [1] [2]. Transaction performed by customer's BUID will transfer to specific financial institute like Banks, Insurance, Shares, Bonds, Post Office, Income tax, Import/Export, Loan etc where it will be happened as per intuitive perception of customer. Further the structure of financial institute like Banks, Insurance, Shares, Bonds, Post Office, Income tax, Import/Export, Loan etc in financial transaction model will have branches[1] [2]. This architecture will help to control and monitor the Income details, Transaction details, tax department and other details of the customers [3]. The data warehouse architecture for financial institute is designed to connect all financial institutes in a network and the data of customers are stored in Data Warehouse through data mart [3]. The channels, financial institutes and customers will access proposed data warehouse for financial institutes.

Data warehouse architecture for financial institutes needed security from illegal access through unauthorized user. Security model will provide security to customers while opening new account using 7 - Tier Architecture Model and making transactions [8]. Security model will identify biometric detail of customer or employees and make allow them for transaction. Security model will provide security for online, mobile, kiosk, green computing and financial institute etc transactions of customers using BUID [4]. The data sources like Bank, Insurances, Shares, Bonds, Post Office, Income Tax, Imp/Exp and Loans data are transfer by ETL process to Data warehouse in respective data marts with several mentioned security levels. The Customers and various channels like income tax department, government accountant and auditors, Government legal regulatory authority, all tax department and overall financial system will access data from data warehouse by executing SQL queries [1]. 


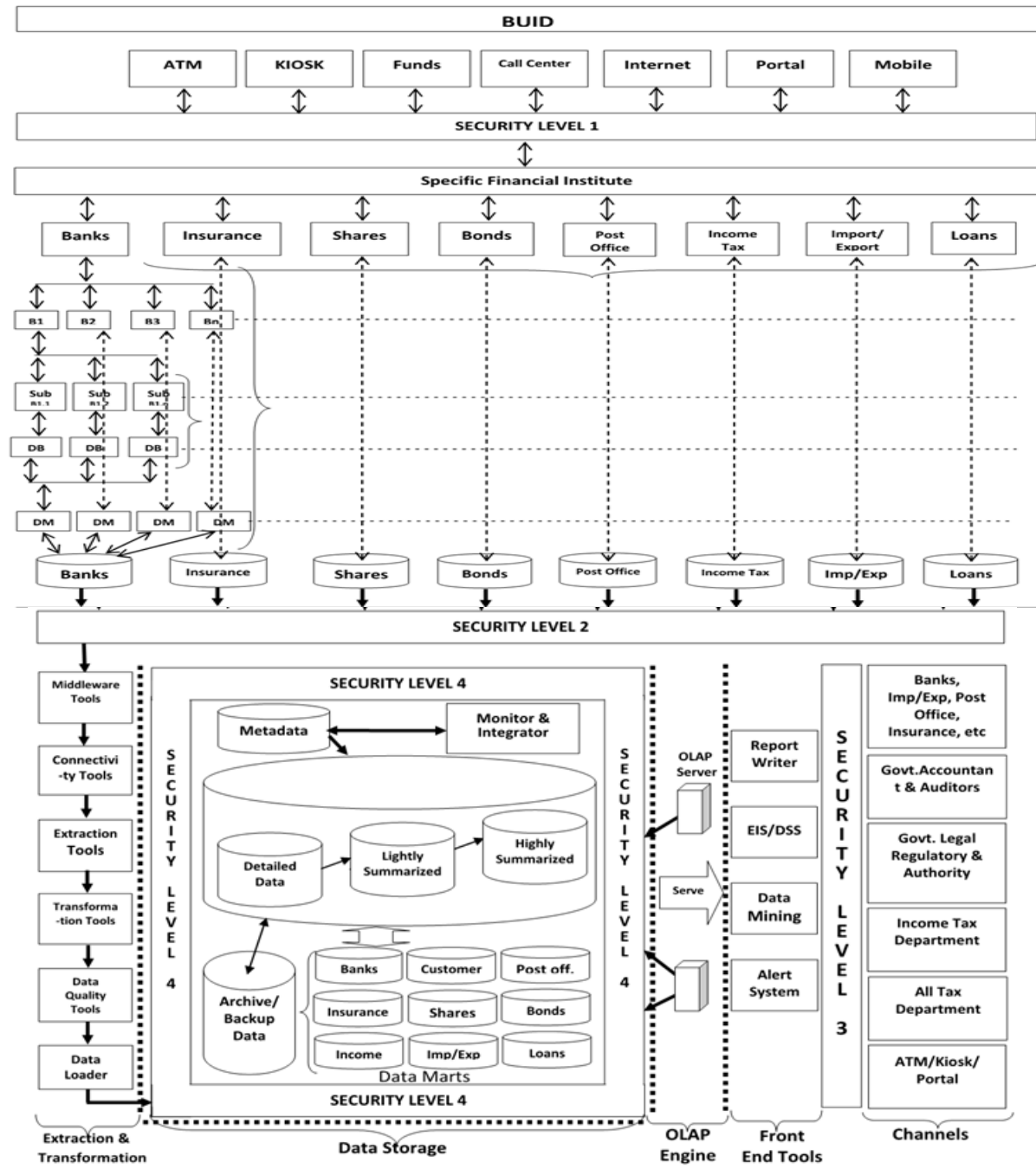

Fig 1: Integrated Data Warehouse for Financial Institutes with Security Levels [1]

\section{Measured Performance Of A Result}

The front end tools of integrated data warehouse for financial institute with security levels included report writer, EIS/DSS, alert system and Data mining. Data query and reporting tools used for deliver warehouse-wide data access through simple interfaces that the SQL language from all financial institute end users through security level 4 [1]. There are several results of proposed architectures. We have shown result of "List of Loan Defaulter" in table1.

\begin{tabular}{|c|c|c|c|c|c|c|c|c|c|}
\hline \\
\hline \multicolumn{10}{|c|}{ Account Number IFSC Code Branch Code Balance Type of Account Type of Loan Amt Sancioned Balance An } \\
\hline 1 & AAAAPA13091957P & 85256369331 & 56478 & 8988 & 50000 & Loan Account & House Loan & 1200000 & 1000000 \\
\hline 2 & AAAAPB10061984K & 55555895623 & 63594 & 8988 & 8000 & Loan Account & Business Lo... & 2000000 & 1000000 \\
\hline 3 & AAAAPBO1011972C & 54612389999 & 56478 & 5689 & 9000 & Loan Account & CarLoan & 400000 & 100000 \\
\hline 4 & AAAAPK24061973... & 85256369741 & 56478 & 8988 & 50000 & Loan Account & House Loan & 1200000 & 1000000 \\
\hline 5 & AAAAPBOBO92012D & 55555444412 & 63594 & 4560 & 8000 & Loan Account & Business Lo... & 2000000 & 1000000 \\
\hline \multicolumn{10}{|c|}{ 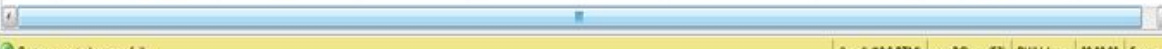 } \\
\hline QQ & 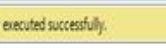 & & & & & & (Aoca) (ADO RTM & 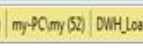 & 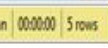 \\
\hline
\end{tabular}

Table 1: List of Loan Defaulter

\subsection{Execution Plan:}

SQL Server Management Studio is an interactive, graphical tool that enables a database administrator or developer to write queries, execute multiple queries simultaneously, view results, analyze the query plan, and receive assistance to improve the query performance. The Execution Plan options graphically display the data retrieval methods chosen by the SQL Server query optimizer. The graphical display is very useful for 
understanding the performance characteristics of a query. There are three query executed in SQL program for Result "List of Loan Defaulter". The following Fig 2: Graphical Execution Plan of Result "List of Loan Defaulter" shows each query in the batch that is analyzed is displayed, including the cost of each query as a percentage of the total cost of the batch.

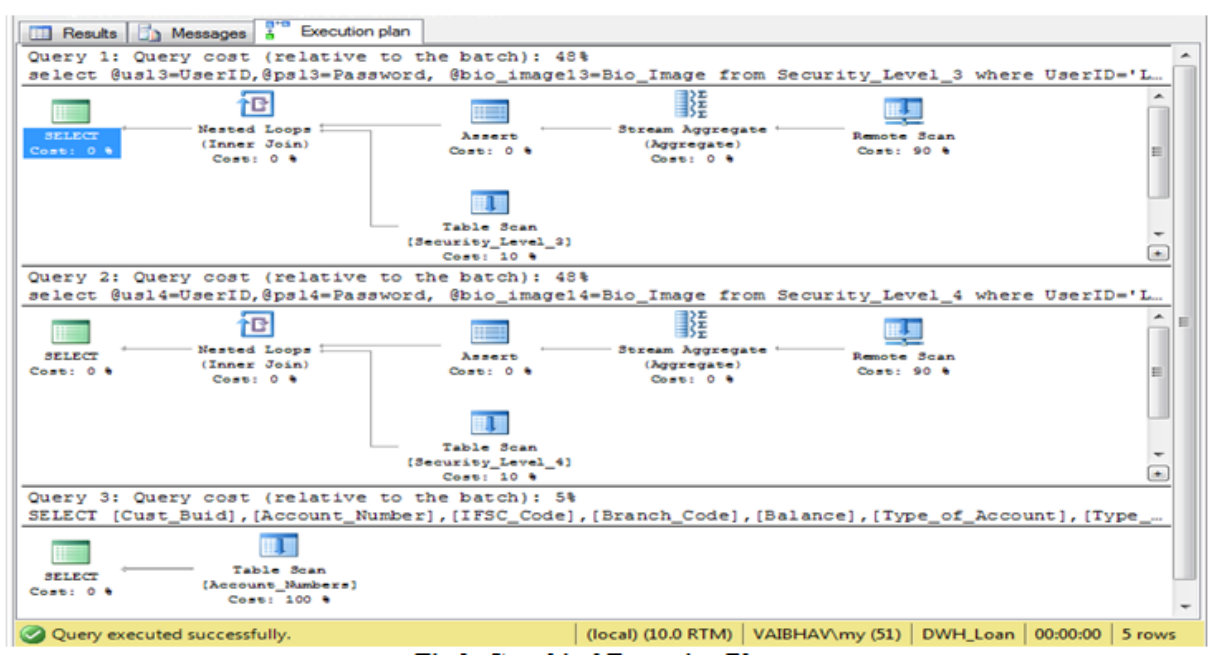

Fig 2: Graphical Execution Plan.

\subsection{Statistical Performance:}

The client statistics is used as Data tools provided by SQL Server to determine performance of query. The client statistics provides the response times for frequently used queries. Such statistics shows client execution time, query profile, packets/bytes sent received, application profile, network, and time statistics etc for the execution. Such metrics allow you to gauge or test the efficiency of script or query. The following Statistical performance is shown for the most recent executions, and they are averaged in milliseconds for Result "List of Loan Defaulter", as shown in Table 2.

\begin{tabular}{|c|c|c|c|c|c|c|}
\hline \multicolumn{7}{|l|}{ 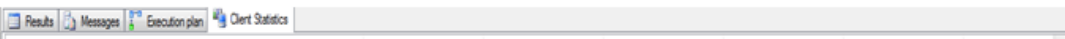 } \\
\hline & Trial 5 & Trial 4 & Trial 3 & Trial 2 & Trial 1 & Average \\
\hline \multicolumn{7}{|l|}{ Query Profile Statistics } \\
\hline Number of INSERT, DELETE and UPDATE stat... & 0 & +0 & $\rightarrow 0$ & $\rightarrow 0$ & $\rightarrow 0$ & $\rightarrow 0.0000$ \\
\hline Rows affected by INSERT, DELETE, or UPDAT... & 0 & $\rightarrow 0$ & $\rightarrow 0$ & +0 & $\rightarrow 0$ & $\rightarrow 0.0000$ \\
\hline Number of SELECT statements & 6 & +6 & +6 & $\rightarrow 6$ & $\rightarrow 6$ & +6.0000 \\
\hline Rows returned by SELECT statements & 10 & +10 & +10 & +10 & $\rightarrow 10$ & +10.0000 \\
\hline Number of transactions & 0 & $\rightarrow 0$ & $\rightarrow 0$ & $\rightarrow 0$ & $\rightarrow 0$ & +0.0000 \\
\hline \multicolumn{7}{|l|}{ Network Statistics } \\
\hline Number of server roundtrips & 3 & +3 & +3 & +3 & $\rightarrow 3$ & $\rightarrow 3.0000$ \\
\hline TDS packets sent from client & 3 & +3 & +3 & +3 & +3 & +3.0000 \\
\hline TDS packets received from server & 12 & +12 & +12 & +12 & $\rightarrow 12$ & $\rightarrow 12.0000$ \\
\hline Bytes sent from client & 2548 & +2548 & $\rightarrow 2548$ & $\rightarrow 2548$ & +2548 & +2548.0000 \\
\hline Bytes received from server & 40669 & +40669 & $\rightarrow 40669$ & $\rightarrow 40669$ & $\downarrow 40671$ & $\rightarrow 40669.4000$ \\
\hline \multicolumn{7}{|l|}{ Time Statistics } \\
\hline Client processing time & 3 & 47 & $\uparrow 3$ & 47 & $\uparrow 3$ & +4.6000 \\
\hline Total execution time & 18 & +19 & $\uparrow 13$ & +30 & $\uparrow 26$ & +21.2000 \\
\hline Wait time on server replies & 15 & $\uparrow 12$ & $\uparrow 10$ & +23 & +23 & $\rightarrow 16.6000$ \\
\hline 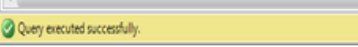 & & & & 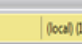 & 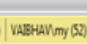 & 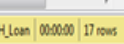 \\
\hline
\end{tabular}

Table 2: Statistical Performance.

\subsection{Query Performance:}

The Activity Monitor which is available in SQL Server 2008 Management Studio is a tool which can be used by database developers and administrators to get a quick overview of SQL Server 2008 system performance. There are many enhancements in Activity Monitor in SQL Server 2008 like a graphical display of Processor Time, Waiting Tasks, Database I/O's, Batch Requests, Processes, Resource Waits, Data File I/O's and also information about the Recent expensive queries. The Recent Expensive Queries section, database administrators can quickly identify poorly performing queries in an SQL Server Instance. The Fig 3 shows information about the most expensive queries that have been run on the instance over the last 30 seconds in which Result "List of Loan Defaulter" queries executed. 
As observed in Recent Expensive Query of Activity Monitor, the Result-1 queries are not sort listed as expensive queries. Hence the Result-1 Queries have given satisfactory performance.

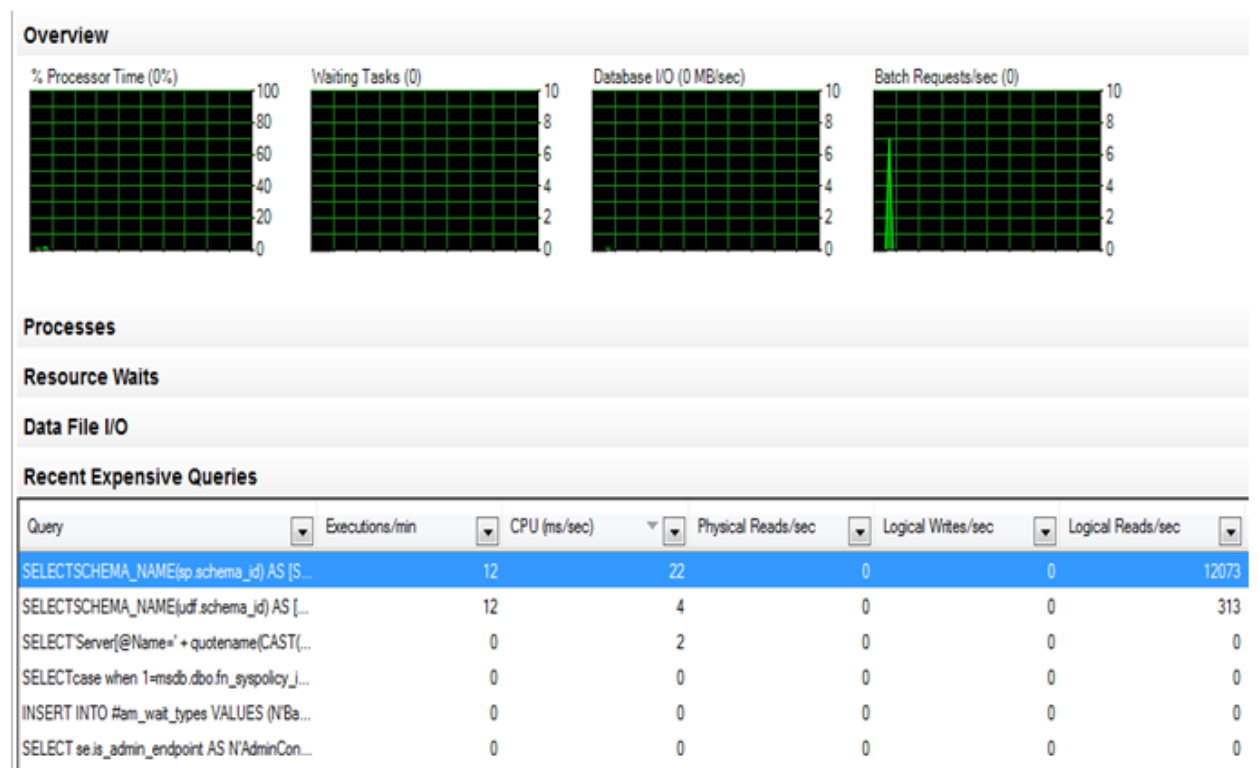

Fig 3: Activity Monitor for Result-1 Queries

\section{Conclusion}

Integrated Data Warehouse for Financial Institutes with Security Levels is a architecture for Indian financial system. The architecture performance has been measure in SQL Server Management Studio by observing results. The Execution Plan options graphically display the data retrieval methods chosen by the SQL Server query optimizer. The client statistics provides the response times for frequently used queries. The most expensive query is run on the instance over the last 30 seconds in which Result "List of Loan Defaulter" queries executed and Observed, the Result-1 Queries have given satisfactory performance.

\section{References}

[1] Vaibhav R Bhedi, Shrinivas P Deshpande and Ujwal A Lanjewar," Secured Integrated Data Warehouse Architecture for Financial Institutes", International Journal of Electronics, Communication \& Soft Computing Science and Engineering (IJECSCSE), Special Issue-ATCON-2015, ISSN: 2277-9477, Impact Factor: 4.5.

[2] Vaibhav R Bhedi. "Design and Study of Financial Transaction Model for BUID". International Journal of Computer Applications (0975 - 8887) ISBN: 973-93-80870-28-,57(13):12-15, November 2012. Published by Foundation of Computer Science, New York, USA, Impact Factor: 0.821.

[3] Vaibhav R Bhedi, Shrinivas P Deshpande and Ujwal A Lanjewar "Data Warehouse Architecture for Financial Institutes to Become Robust Integrated Core Financial System using BUID”. International Journal of Advanced Research in Computer and Communication Engineering Vol. 3, Issue 3, March 2014. ISSN (Online) : 2278-102, ISSN (Print) : 2319-5940, Impact Factor: 1.770 .

[4] Vaibhav R Bhedi, Shrinivas P Deshpande and Ujwal A Lanjewar, "Design and Study of Security Model for Core Financial System". International Journal of Emerging Trends \& Technology in Computer Science (IJETTCS) Volume 3, Issue 6, NovemberDecember 2014.ISSN: 2278-6856, Impact Factor:3.258.

[5] Shrinivas P. Deshpande, Vaibhav R. Bhedi, Ujwal A. Lanjewar, Sandeep R. Sirsat, "Simulation of Secured Integrated Data warehouse Model for Financial Institute “, $2^{\text {nd }}$ International Conference on Web Research, University of Science and Culture, Tehran, Iran, 27, 28 April 2016, Paper No:48.

[6] Ghezzi, Carlo, Mehdi Jazayeri, and Dino Mandrioli. Fundamentals of software engineering. Second Edition, Prentice Hall PTR, pp. 17-20, ISBN: 013099183X, 2009.

[7] Senn, James A. Analysis and design of information systems. , Second Edition,McGraw-Hill, Inc., pp.718, 386, ISBN: 0070562210, 1990

[8] Vaibhav R Bhedi, Ujwal A Lanjewar and Shrinivas P Deshpande, "Design and Study of 7 - Tier Architecture Model for Opening Account in Present Financial System". International Journal of Computer Applications (0975 - 8887) 37(3):25-29, January 2012. Published by Foundation of Computer Science, New York, USA, Impact Factor: 0.821. 